\title{
The Disproportionate Impact of Hypertensive Cardiovascular Disease in African Americans: Getting to the Heart of the Issue
}

\author{
Kenneth A. Jamerson, MD
}

The prevalence of hypertension in African Americans is among the highest in the world. Persons in this group develop hypertension at a younger age than non-African Americans and develop more severe complications, including stroke, cardiovascular disease, and renal failure. The factors that impart this high risk to this population remain poorly understood and, undoubtedly, environmental factors overshadow genetic predisposition. While identifying the pathophysiologic and environmental factors that contribute to ethnic disparity in disease is important, finding a long-term solution is crucial. Steps that can have an important impact on health outcomes of African Americans are presently available. Awareness of ethnicity as a risk factor for hypertension can allow health care providers to identify persons who are likely to benefit most from early, aggressive intervention. Modifiable factors such as smoking, diet, and sedentary lifestyle, as well as undertreatment of hypertension by physicians, can be targeted immediately. (J Clin Hypertens. 2004;6(4 suppl 1):4-10)

${ }^{\circ} 2004$ Le Jacq Communications, Inc.

From the Department of Internal Medicine, University of Michigan Health Systems, Ann Arbor, MI Address for correspondence:

Kenneth A. Jamerson, MD, Department of Internal Medicine, University of Michigan, 3918 Taubman Center, Box 0356, Ann Arbor, MI 48109-0356

E-mail:jamerson@umich.edu

www.lejacq.com

ID: 3563
$T^{1}$ he need for effective interventions to reduce the incidence of cardiovascular disease and end organ damage in the African-American community is apparent from reviewing available statistics. It was sobering when McCord et al. ${ }^{1}$ published a comparison of life expectancies in the United States and Bangladesh revealing that an adult man living in Harlem, a largely AfricanAmerican community in New York, NY, had a $45 \%$ greater risk of dying before age 65 years than his counterpart in Bangladesh, one of the poorest nations in the world. At that time, the same adult African-American man had a $92 \%$ greater risk of premature death than a white man in the United States. ${ }^{1}$

Since the McCord study was conducted, the life expectancy of African Americans has steadily risen from 68.1 years to 71.7 years, a 5\% rate increase in life expectancy that is, in fact, greater for African Americans than whites (Figure 1). Despite these gains, a significant life expectancy gap remains in the United States today-African Americans can expect to die almost 6 years earlier than non-African Americans. ${ }^{2}$

Cardiovascular disease is by far the most common cause of death among African Americans, accounting for $>27 \%$ of total deaths (Figure 2). Stroke and diabetes account for an additional $6.7 \%$ and $4.2 \%$ of deaths, respectively. ${ }^{2}$ However, African Americans in the United States are also more likely to die than whites from illnesses such as human immunodeficiency virus and as the result of accidents or homicides $(2.7 \%$ vs. $0.3 \%, 4.3 \%$ vs. $4.0 \%$, and $2.8 \%$ vs. $0.4 \%$, respectively). ${ }^{2}$ 


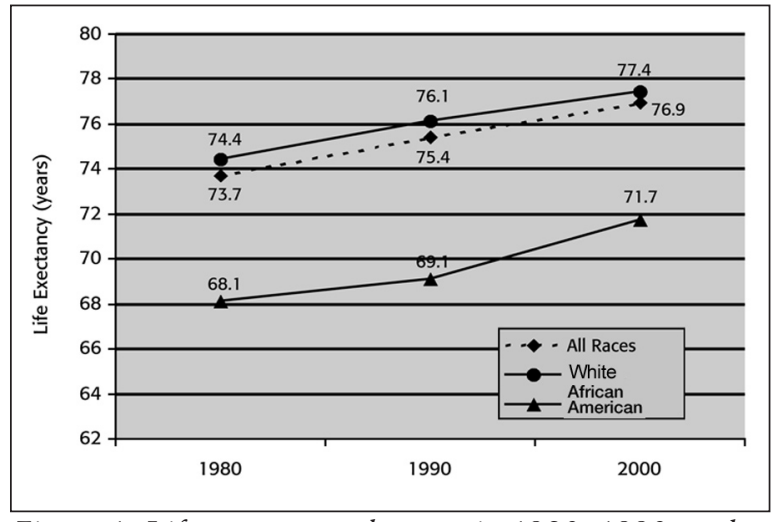

Figure 1. Life expectancy by race in 1980, 1990, and 2000. Source: Nat Vital Stat Rep. 2002;50:1-85. ${ }^{2}$

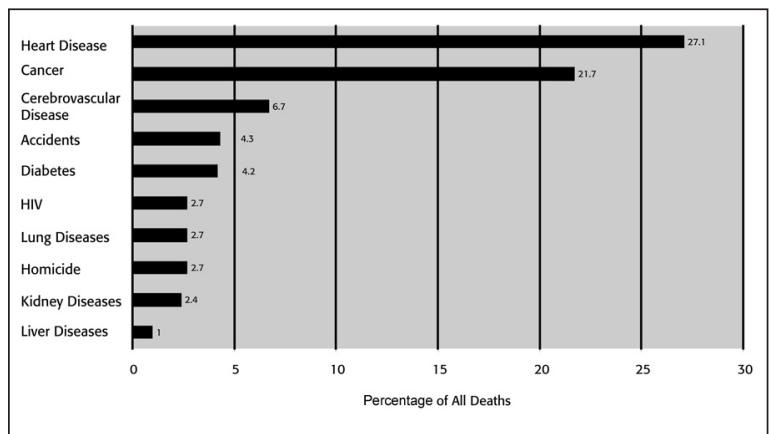

Figure 2. Leading causes of death and percentage of all deaths among African Americans

Source: Nat Vital Stat Rep. 2002;50:1-85. ${ }^{2}$

In light of the statistics for cardiovascular disease and stroke, it is not surprising that African Americans have higher rates of hypertension than whites or Mexican Americans (Figure 3). ${ }^{3}$ Hypertension is more prevalent and develops at a younger age in African Americans. Complications of uncontrolled hypertension, including stroke, left ventricular hypertrophy, heart failure, myocardial infarction, and end-stage renal disease are also more common. Overall, $80 \%$ of this population can expect to reach hypertension-blood pressure $\geq 140 / 90 \mathrm{~mm} \mathrm{Hg}$ as described in the Seventh Report of the Joint National Committee on Prevention, Detection, Evaluation, and Treatment of High Blood Pressure (JNC 7). ${ }^{4}$

\section{IS THERE A UNIQUE ETIOLOGY FOR HYPERTENSION IN AFRICAN AMERICANS?}

A number of studies have compared the pathophysiology of hypertension in African Americans and whites. Expanded plasma volume, prematurely decreased renal function, increased vascular reactivity and salt sensitivity, and reduced plasma renin activity have been recurrent themes in many of these studies. Although these factors may contribute to the higher incidence of hypertension among African Americans, it is unlikely that any of the proposed differences account for much of the ethnic disparities. Moreover, these pathophysiologic features represent quantitative differences in both African-American and non-African-American hypertensives, rather than unique qualitative differences among African Americans.

Of the 17 published studies examining the contribution of plasma volume in hypertension between African Americans and whites, one is particularly noteworthy. In this study, 172 consecutive patients were evaluated. Twice as many African-American patients $(30 \%)$ had expanded plasma volume compared with whites (15\%). However, almost $70 \%$ of African Americans had either normal or contracted plasma volume. Hence, expanded plasma volume represents a pathophysiologic phenomenon in only minority of African Americans. Importantly, the preferred standard strategy of using diuretic therapy to treat the expanded volume in African Americans may not be relevant to $70 \%$ of persons in this group. ${ }^{5}$

Another interesting set of findings emerged from a study undertaken at the University of Michigan. This study compared plasma renin activity in African-American and white medical students following a high-salt (pizza) or a low-salt $(20 \mathrm{mEq})$ diet. African Americans had a much lower plasma renin activity at baseline; upon withdrawing salt, the plasma renin activity in the African-American students began to rise at a rate faster than that of whites (Figure 4). This observation raises the question of whether the low renin activity observed in African Americans is more a function of dietary patterns (salt) than a specific genotype.

The findings of the Dietary Approaches to Stop Hypertension (DASH) subgroup analysis in African Americans also support a role for dietary manipulation in this group of patients with hypertension. ${ }^{6}$ At the end of 8 weeks of dietary intervention, blood pressure reduction for participants eating a combination diet rich in potassium, magnesium, and calcium and low in cholesterol was compared with that of participants eating a control diet. Both diets contained $3000 \mathrm{mg} / \mathrm{d}$ sodium. ${ }^{6}$ Blood pressure reduction in African-American participants on the combination diet was $-6.9 /-3.7 \mathrm{~mm} \mathrm{Hg}$, almost twice the reduction observed in whites (Figure 5). ${ }^{6}$ These findings can be inferred to suggest that the dietary pattern in African Americans contributes to higher blood pressure in this patient group. 
To determine whether a higher prevalence of hypertension exists in different groups of African origin, Cooper et al. ${ }^{7}$ analyzed seven populations of African origin including African Americans, West Africans, and Caribbeans of African descent. The prevalence of hypertension rose from $10 \%$ to $15 \%$ of persons in Africa, to $20 \%-25 \%$ in the Caribbean, and to $33 \%$ in the United States. ${ }^{7,8}$ Obesity and type 2 diabetes also followed this same geographical gradient for increased prevalence of hypertension, suggesting that environmental factors that affect weight gain are also predisposing factors to the development of hypertension (Figure 6). ${ }^{9}$ Similar observations have been made for Asians residing on Pacific islands and in the United States compared with their native lands, suggesting a Westernizing effect that may be related to diet and exercise patterns.

Even within the United States, regional differences in the incidence of stroke, myocardial infarction, and end-stage renal disease (all complications associated with hypertension) have been reported for African Americans and non-African Americans. ${ }^{10,11}$ In fact, in a 10 -state area in the southeastern region of the United States known as the Stroke Belt, stroke mortality rates are $10 \%$ higher than the mean rate for the rest of the country. After controlling for race, patients residing within the Stroke Belt had higher values for blood pressure, body mass index, urinary sodium excretion, and serum creatinine. On average, they had larger left ventricular mass and lower serum potassium levels, alcohol intake, and plasma renin activity. African Americans had higher diastolic blood pressure (DBP), serum creatinine levels, and smoking rates and lower household incomes, medication adherence rates, 24-hour urinary sodium and potassium excretion, serum potassium and fasting glucose levels, alcohol intake, and plasma renin activity than whites. Plasma renin activity, obesity, fasting glucose levels, alcohol intake, and estimated household income were not predictive of response to any of the study medications. These results suggest that environmental and/or other factors may contribute to these geographic and racial differences in stroke mortality and to the limited success with antihypertensive monotherapy. ${ }^{10}$

Lackland et al. ${ }^{12}$ took these observations one step further and found a graded risk of stroke by birthplace among African Americans and non-African Americans living in South Carolina. The proportional mortality ratios were highest for persons born in South Carolina, followed by those born elsewhere in the Stroke Belt. Persons who moved to

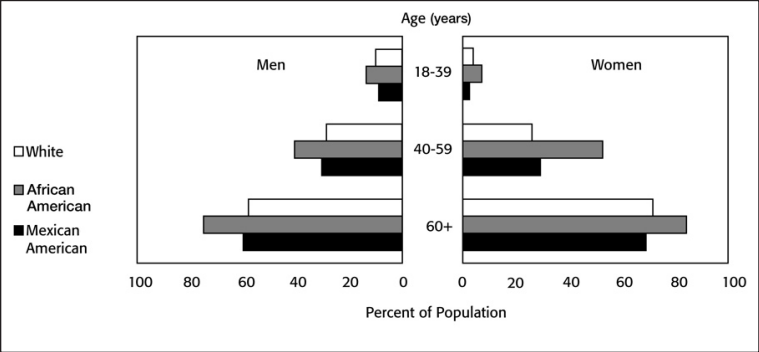

Figure 3. Percentage of the adult population with bypertension for 1999-2000 by gender and race. Those persons included had systolic blood pressure $>140 \mathrm{~mm} \mathrm{Hg}$ or diastolic blood pressure $>90 \mathrm{~mm} \mathrm{Hg}$ or were on antibypertensive medication

Adapted with permission from Disease statistics. In: NHLBI Fiscal Year 2002 Fact Book. Bethesda, MD: National Institutes of Health, National Heart, Lung, and Blood Institute; 2003:35-54.

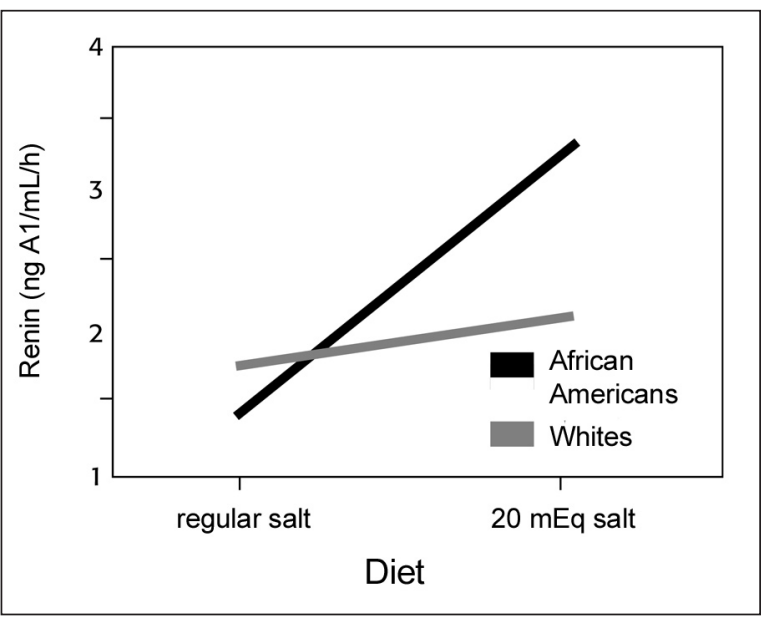

Figure 4. Plasma renin activity following high-salt or low-salt intake

Source: K. Jamerson, unpublished data.

South Carolina after being born outside the Stroke Belt had the lowest proportional mortality ratios. The disparities for birthplace and subsequent stroke mortality were greatest for African Americans. ${ }^{12}$

Clearly, environmental factors influence risk of hypertension, related complications, and response rates to antihypertensive therapy. However, comparisons between African Americans and Hispanics reflect the complexity of causal factors underlying population-group differences. While Hispanics and African Americans share a similar prevalence of obesity, type 2 diabetes, and socioeconomic disadvantage compared with whites, Hispanics have a lower all-cause death rate than African Americans (330/100,000 compared with 810/100,000 for African Americans) and a lower incidence of cardiovascular or stroke death (Figure 5). ${ }^{2,3}$ Unfortunately, the lack of a simple answer to explain the racial differences in risk 


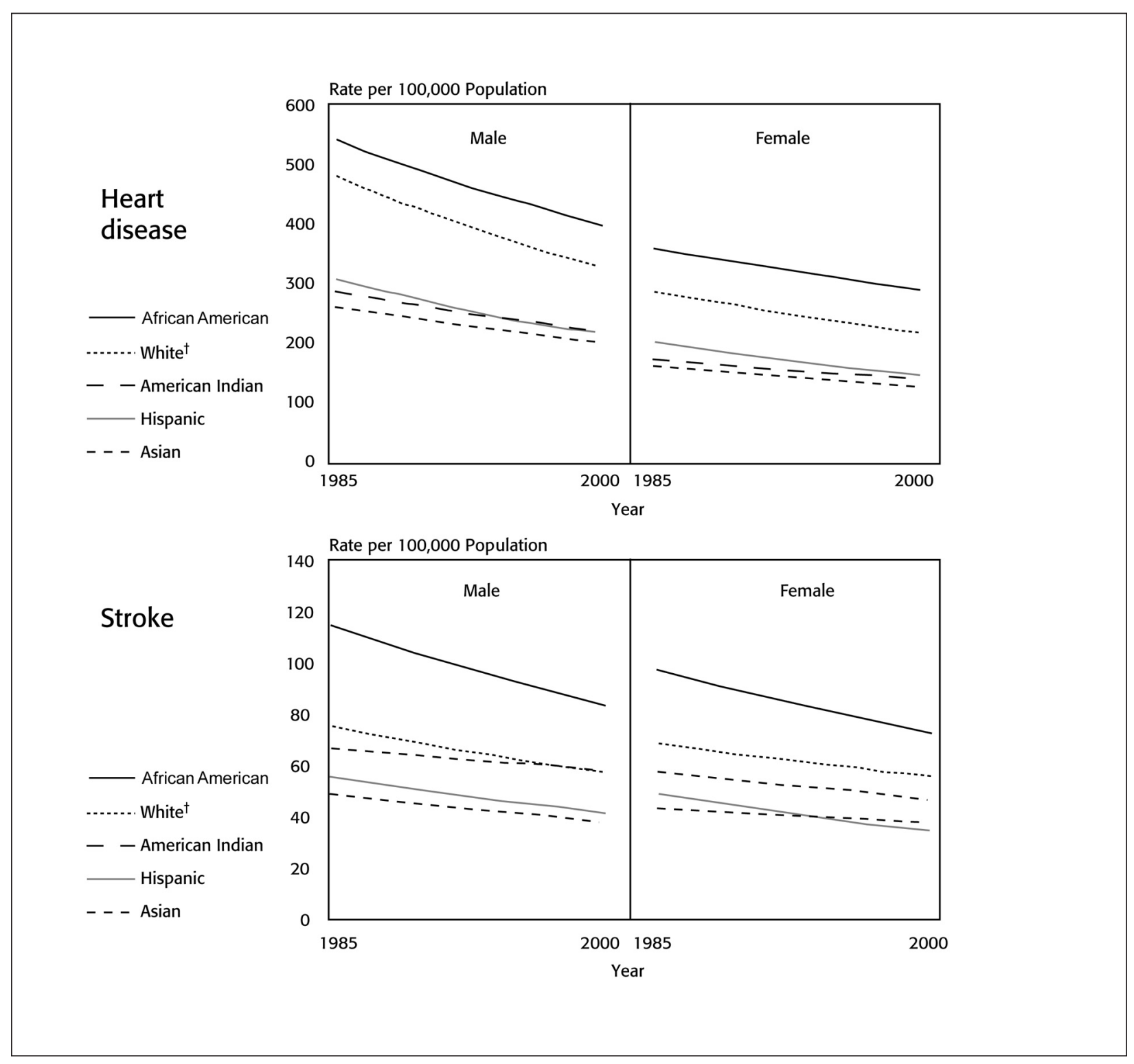

Figure 5. Age-adjusted death rates for heart disease and stroke by gender, race, and ethnicity, United States 19852000. Each line is a log-linear regression derived from the actual rates; ${ }^{\dagger}$ non-Hispanic

Adapted with permission from Disease statistics. In: NHLBI Fiscal Year 2002 Fact Book. Bethesda, MD: National Institutes of Health, National Heart, Lung, and Blood Institute; 2003:35-54.

has contributed to the idea that treating African Americans may be difficult.

\section{DO AFRICAN AMERICANS \\ RESPOND DIFFERENTLY TO \\ MEDICAL INTERVENTION?}

Treatment of hypertension in African Americans is critical to reducing the burden of cardiovascular disease by preventing target organ damage, including heart failure, stroke, and renal failure. Underdiagnosed, untreated, and inadequately treated hypertension has had a devastating effect on this population. The failure of health care pro- viders to treat hypertension early and to aggressively pursue target blood pressures may be, in part, a result of the misconception that African Americans are less responsive to medical interventions than other racial or ethnic groups. This belief may, in turn, be related to the underrepresentation of African Americans in many large clinical trials.

The Department of Veterans Affairs Cooperative Study Group on Antihypertensive Agents evaluated single-drug therapy with six antihypertensive drugs in a titration-to-effect study in 1292 men. ${ }^{13}$ The primary outcome measure was the percentage of randomized patients who reached the blood 
pressure goal $(\mathrm{DBP}<90 \mathrm{~mm} \mathrm{Hg})$ during the 4- to 8 -week titration period and maintained adequate blood pressure control (<95 mm Hg DBP) for 1 year. Response rates differed for age and race. Nonetheless, as many as $64 \%$ of African-American patients achieved adequate blood pressure control on monotherapy. Diltiazem ranked first for both older ( $\geq 65$ years) and younger African Americans with $64 \%$ responding in both age groups. Captopril was the least efficacious in older and younger African Americans (response rates of $20 \%$ and $32 \%$, respectively). ${ }^{13}$

The Losartan Intervention For Endpoint (LIFE) trial may have important implications for the African-American community. The LIFE trial compared the efficacy of an angiotensin-receptor blocker-based treatment regimen (losartan) with a treatment program based on a $\beta$ blocker (atenolol) for reducing the incidence of a composite end point of death, myocardial infarction, or stroke in patients with hypertension with left ventricular hypertrophy as determined by electrocardiographic criteria. ${ }^{14}$ In the overall population $(\mathrm{N}=9193)$ the losartan-based regimen significantly reduced the risk of reaching the composite end point by $13 \%(p=0.021)$ and the risk of stroke by $25 \% \quad(p=0.001)$ compared with the atenolol-based regimen. ${ }^{14}$ However, a retrospective subgroup analysis of the data for the $533(6 \%)$ African-American patients enrolled in LIFE suggested that African Americans receiving a losartan-based regimen had a higher risk of cardiovascular death, myocardial infarction, or stroke $(46 / 270[17 \%]$ for losartan vs. $29 / 263[11 \%]$ for atenolol). This finding occurred despite the fact that African Americans achieved similar levels of blood pressure control compared with whites. Moreover, losartan was superior to atenolol in regressing left ventricular hypertrophy in the African-American cohort. ${ }^{15}$ While losartan was approved by the US Food and Drug Administration ${ }^{16}$ to reduce the risk of stroke in patients with hypertension and left ventricular hypertrophy based on the LIFE trial data, the new labeling includes the unprecedented exclusion of efficacy regarding the use of this drug in African Americans.

Certainly, more investigation into the efficacy of angiotensin-receptor blocker treatment in African Americans is warranted for this indication. However, the wisdom of excluding a particular subgroup from the indication based on results that defy pathophysiologic explanation is questionable. Instead, prescribing losartan or other agents that block the renin-angiotensin system should be based on individual patient needs to significantly lower

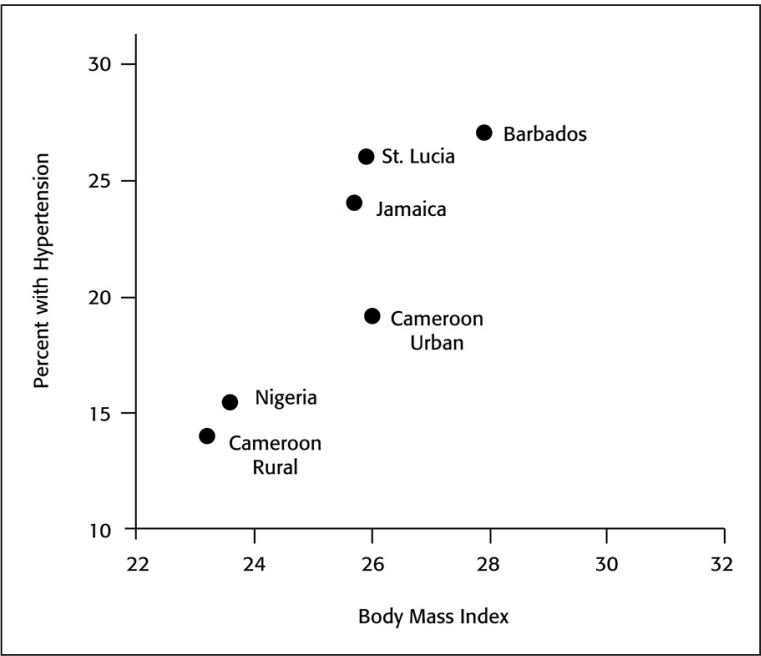

Figure 6. Geographic differences in the prevalence of bypertension in populations of African origin Adapted from Am J Hypertens. 1997;10:804-812. ${ }^{7}$

risk of cardiovascular death, myocardial infarction, or stroke in the overall population.

Inadequate sampling of African Americans (as in LIFE) can yield confusing and problematic results. In addition to inadequate numbers of African Americans enrolled in many large clinical trials, the interpretation of data must take into account differences in physician prescribing habits in treating African Americans and whites. Several large clinical trials have demonstrated that monotherapy is inadequate for long-term blood pressure control and that in many instances between two and four antihypertensive agents are required to adequately control uncomplicated hypertension regardless of race. ${ }^{17-20}$ Therefore, trial designs that allow study drug titration-to-effect dosing and the use of additional antihypertensive agents as needed most closely mimic the real world.

The Antihypertensive and Lipid-Lowering Treatment to Prevent Heart Attack Trial (ALLHAT) was such a trial and, in addition, had an over sampling of African Americans (one third) in the overall study population. In the total population, amlodipine, chlorthalidone, and lisinopril were equally effective in reducing the risk of the primary combined outcome of fatal coronary heart disease or nonfatal myocardial infarction. ${ }^{18}$

However, African Americans were somewhat less likely to achieve blood pressure control than non-African Americans, especially those assigned to the ACE inhibitor arm of the study. At the end of 5 years, $59 \%$ of African-American women and $63 \%$ of African-American men had blood pressure 


\begin{tabular}{lccc|}
\hline \multicolumn{4}{l}{ Table. Patient Characteristics at Baseline and 2 Years Following Intervention in a Community-Based Trial } \\
\hline Characteristic & BASEline & After Intervention & Change From Baseline \\
\hline Weight (lb) & 219 & 208 & -11 \\
BMI & 35.8 & 34 & -1.8 \\
SBP (mm Hg) & 129 & 123 & -6 \\
DBP (mm Hg) & 82 & 76 & -6 \\
Cholesterol (mg/dL) & 196 & 190 & -6 \\
HDL cholesterol (mg/dL) & 56 & 49 & -7 \\
BMI=body mass index; DBP=diastolic blood pressure; HDL=high-density lipoprotein; SBP=systolic blood pressure \\
Source: K. Jamerson, unpublished data
\end{tabular}

$<140 / 90 \mathrm{~mm} \mathrm{Hg}$. In contrast, the percentage of white patients with controlled blood pressure at the end of 5 years was $65 \%$ and $70 \%$ for women and men, respectively. ${ }^{21}$ It is of particular significance that while an average of two drugs was needed to achieve blood pressure control in the overall population, a multiple logistics analysis determined that the relative odds of being on two or more drugs at the end of the third year of the trial was 0.89 (95\% confidence interval, 0.84-0.94) for African Americans compared with whites. This result suggests that African Americans may not have been treated as aggressively as non-African Americans. ${ }^{21}$ In this study that included a more than adequate representation of African Americans, the interpretation of the results may be confounded by a possible disparity in treatment.

A study that is elaborated on in the article by Saunders ${ }^{22}$ was designed to answer the question of optimal treatment for African Americans with renal insufficiency. The African American Study of Kidney Disease and Hypertension (AASK) used multiple pharmacotherapeutic strategies to control blood pressure in the hopes of slowing renal disease progression in an exclusively AfricanAmerican cohort. At the end of the study, the mean systolic blood pressure/DBP was $135 / 82 \mathrm{~mm} \mathrm{Hg}$ for those in the ACE inhibitor group and 135/81 $\mathrm{mm} \mathrm{Hg}$ for those in the calcium channel blocker group. The average number of drugs required to achieve these blood pressure reductions was 2.75 for both treatment groups. ${ }^{23}$ Making comparisons within an all-African-American cohort is useful in that it eliminates subtle or not so subtle ethnic biases in treatment and reduces the impact of dietary and cultural differences otherwise found in cross-cultural studies. The AASK study results aptly document that ideal blood pressure control can be achieved regardless of the selection for monotherapy and the importance of renin-angiotensin system blockade in target organ protection in African Americans.

\section{WHAT STRATEGIES WORK BEST IN EDUCATING AFRICAN AMERICANS?}

Successful strategies for reaching the AfricanAmerican community and effecting healthful lifestyle changes are both labor intensive and expensive. However, the payoff can be substantial if initial successes can be maintained. At the University of Michigan Health Care System, a community-based partnership has been developed and successfully translated into an initiative to educate the African-American community about cardiovascular disease as well as to achieve weight and blood pressure reductions.

In initiating this trial, the need to understand the concerns of this community beyond the scope of cardiovascular health became apparent early on. After sponsoring focus groups, developing community task groups to investigate solutions to community issues, and holding community events of interest to various age groups, 112 subjects with an average age of 49 years were enrolled in a program designed to reduce cardiovascular risk. Women comprised $54 \%$ of the cohort and $30 \%$ of this study population had hypertension at the time of enrollment.

The program relied heavily on community involvement. All activities occurred in the community setting: community health care leaders monitored progress and church leaders reinforced the efforts of participants. The University of Michigan Health Care System provided counseling support by dietitians and trainers. Participants were offered the American Heart Association diet as well as dietetic support, provided with the opportunity to exercise, and were taught mind-body techniques. After more than 2 years and $\$ 6000$ per patient, weight, body mass index, and blood pressure had all been reduced (Table). After 30 months, most patients continue to maintain these changes. A similarly labor-intensive diabetes control and complications trial also found a persistent effect of the intervention with differences in behavior and goal attainment evident years after the study end. 


\section{CONCLUSIONS}

Hypertension is more prevalent, occurs at an earlier age, and leads to more end organ damage in African Americans than in other Americans. Genetic differences, which have been invoked to explain these differences, may play a minor role. However, environmental factors are important. Whatever the pathophysiology behind ethnicity disease-state disparity, blood pressure can still be successfully controlled in this population using a variety of agents currently available.

To reduce the disproportionate burden of cardiovascular disease among African Americans, patients and health care providers need to first understand the risks. Physicians need to use that knowledge to provide early, aggressive intervention. Community-based public health initiatives are a first step and can be used to achieve weight loss and blood pressure reduction.

\section{REFERENCES}

1 McCord C, Freeman HP. Excess mortality in Harlem. N Engl J Med. 1990;322:173-177.

2 Anderson RN. Deaths: leading causes for 2000. Natl Vital Stat Rep. 2002;50:1-85.

3 Morbidity and Mortality: 2002 Chartbook on Cardiovascular, Lung, and Blood Diseases. Bethesda, MD: National Institutes of Health; 2002.

4 Chobanian AV, Bakris GL, Black HR, et al. The Seventh Report of The Joint National Committee on Prevention, Detection, Evaluation, and Treatment of High Blood Pressure: the JNC 7 report. JAMA. 2003;289:2560-2572.

5 Chrysant SG, Danisa K, Kem DC, et al. Racial differences in pressure, volume and renin interrelationships in essential hypertension. Hypertension. 1979;1:136-141.

6 Svetkey LP, Simons-Morton D, Vollmer WM, et al. Effects of dietary patterns on blood pressure: subgroup analysis of the Dietary Approaches to Stop Hypertension (DASH) randomized clinical trial. Arch Intern Med. 1999;159:285-293.

7 Cooper R, Rotimi C. Hypertension in blacks. Am J Hypertens. 1997;10:804-812.

8 Cooper RS, Rotimi CN, Kaufman JS, et al. Prevalence of NIDDM among populations of the African diaspora. Diabetes Care. 1997;20:343-348.

9 Cooper R, Rotimi C, Ataman S, et al. The prevalence of hypertension in seven populations of west African origin. Am J Public Health. 1997;87:160-168.

10 Cushman WC, Reda DJ, Perry HM, et al. Regional and racial differences in response to antihypertensive medication use in a randomized controlled trial of men with hypertension in the United States. Department of Veterans Affairs Cooperative Study Group on Antihypertensive Agents. Arch Intern Med. 2000;160:825-831.

11 Roccella EJ, Lenfant C. Regional and racial differences among stroke victims in the United States. Clin Cardiol. 1989;12:IV18-IV22.

12 Lackland DT, Egan BM, Jones PJ. Impact of nativity and race on "Stroke Belt" mortality. Hypertension. 1999;34:57-62.

13 Materson BJ, Reda DJ, Cushman WC, et al. Single-drug therapy for hypertension in men. A comparison of six antihypertensive agents with placebo. The Department of Veterans Affairs Cooperative Study Group on Antihypertensive Agents. N Engl J Med. 1993;328:914-921.

14 Dahlof B, Devereux RB, Kjeldsen SE, et al. Cardiovascular morbidity and mortality in the Losartan Intervention For Endpoint reduction in hypertension study (LIFE): a randomised trial against atenolol. Lancet. 2002;359:995-1003.

15 Julius S. The LIFE study: special implications for African Americans. Presented at: 2002 International Society on Hypertension in Blacks Annual Meeting; June 8-12, 2002; Miami, FL.

16 Fleming T, Borer J, Armstrong PW, et al. Food and Drug Administration: Cardiovascular and Renal Drugs Advisory Committee, 98th meeting, January 6th-7th, 2003. Circulation. 2003;107:e9002-e9003.

17 Wright JT, Bakris G, Greene T. Effect of blood pressure lowering and antihypertensive drug class on progression of hypertensive kidney disease. Results from the AASK trial. JAMA. 2002;288:2421-2431.

18 ALLHAT Collaborative Research Group. Major outcomes in moderately hypercholesterolemic, hypertensive patients randomized to pravastatin vs usual care: The Antihypertensive and Lipid-Lowering Treatment to Prevent Heart Attack Trial (ALLHAT-LLT). JAMA. 2002;288:2998-3007.

19 Douglas JG, Bakris GL, Epstein M, et al. Management of high blood pressure in African Americans: consensus statement of the Hypertension in African Americans Working Group of the International Society on Hypertension in Blacks. Arch Intern Med. 2003;163:525-541.

20 SHEP Cooperative Research Group. Prevention of stroke by antihypertensive drug treatment in older persons with isolated systolic hypertension. Final results of the Systolic Hypertension in the Elderly Program (SHEP). SHEP Cooperative Research Group. JAMA. 1991;265:3255-3264.

21 Cushman WC, Ford CE, Cutler JA, et al. Success and predictors of blood pressure control in diverse North American settings: the antihypertensive and lipid-lowering treatment to prevent heart attack trial (ALLHAT). I Clin Hypertens (Greenwich). 2002;4:393-405.

22 Saunders E. Managing hypertension in African-American patients. J Clin Hypertens (Greenwich). 2004;6(4 suppl 1):19-25.

23 Agodoa LY, Appel L, Bakris GL, et al. Effect of ramipril vs amlodipine on renal outcomes in hypertensive nephrosclerosis: a randomized controlled trial. JAMA. 2001;285:2719-2728. 